\title{
Philosophiques
}

\section{Goddard, Jean-Christophe, La philosophie fichtéenne de la vie : le transcendantal et le pathologique, Paris, Vrin (coll. " Histoire de la philosophie — Temps modernes »), 1999, 239 pages.}

\section{Manuel Roy}

Volume 29, numéro 1, printemps 2002

Spinoza sous le prisme de son anthropologie

URI : https://id.erudit.org/iderudit/009572ar

DOI : https://doi.org/10.7202/009572ar

Aller au sommaire du numéro

Éditeur(s)

Société de philosophie du Québec

ISSN

0316-2923 (imprimé)

1492-1391 (numérique)

Découvrir la revue

Citer ce compte rendu

Roy, M. (2002). Compte rendu de [Goddard, Jean-Christophe, La philosophie fichtéenne de la vie : le transcendantal et le pathologique, Paris, Vrin (coll. « Histoire de la philosophie - Temps modernes »), 1999, 239 pages.]

Philosophiques, 29(1), 153-155. https://doi.org/10.7202/009572ar d'utilisation que vous pouvez consulter en ligne.

https://apropos.erudit.org/fr/usagers/politique-dutilisation/ 
sa relation primaire au milieu. L'homme imageant et parlant sans cesse de voir les choses directement : il les voit à travers l'écran de représentations qui se sont mises à le posséder de par ses propres transactions antérieures avec des objets et qui sont évoquées par le contenu perceptif présent, l'imprégnant de leur charge symbolique, et s'ajoutant à celle-ci en raison de la nouvelle expérience elle-même. (p. 192)

C'est donc le mérite de cette philosophie unitaire de la vie de trouver à dire l'autotrenscendance de la vie, qui est esprit et liberté, ne serait-ce que dans une ultime version de la philosophie de la vie.

KIM SANG ONG-VAN-CUNG

Université de Poitiers

\section{Goddard, Jean-Christophe, La philosophie fichtéenne de la vie: le transcendantal et le pathologique, Paris, Vrin (coll. « Histoire de la philosophie - Temps modernes »), 1999, 239 pages.}

Les commentateurs ont souvent soutenu la thèse suivant laquelle le projet fichtéen de la Doctrine de la science serait de nature essentiellement épistémologique. Cette lecture s'en tient toutefois à la lettre du fichtéanisme, qu'elle ne fait que répéter sans la comprendre. Pour accéder à l'esprit de la Doctrine de la science, il faut poser la question du sens de l'intérêt du philosophe à l'égard de la question du fondement de la possibilité de la science en général. Sur cette question, le livre de Goddard offre une lumière nouvelle. Loin de trouver son origine dans des préoccupations d'ordre épistémologique, dit Goddard, le projet fichtéen de la Doctrine de la science ressortit avant tout au domaine religieux. En effet, le "principe méthodologique » (p. 19) qui guide la réflexion fichtéenne, écrit-il, est celui-ci : penser n'est pas vivre. Si simple qu'il puisse paraître, ce principe méthodologique n'est selon lui ni plus ni moins que le mot d'ordre qui fonde l'attitude critique dont la philosophie transcendantale est le produit théorique. Cette attitude consiste à résister à la tentation de nier la distinction qui existe entre la pensée et la vie, c'est-à-dire entre le sujet et l'objet, tentation à laquelle cède le dogmatique (le dogmatique de type réaliste en réduisant la pensée à la vie (le sujet à l'objet), le dogmatique de type idéaliste en réduisant la vie à la pensée (l'objet au sujet)). Cette tentation a son origine dans le désir de saisir conceptuellement le point d'unification de la pensée et de la vie, c'est-à-dire dans le désir du bonheur que conditionne cette saisie. Or Dieu est ce point d'unification (chez le réaliste, Dieu est en effet cause première, chez l'idéaliste, il est substance subjective). Le philosophe critique nie donc la possibilité du bonheur parce qu'il nie la possibilité de la connaissance de Dieu. L'attitude critique est donc une attitude essentiellement religieuse : elle consiste à admettre que rien n'est au-delà de Dieu, que Dieu ne peut être connu, mais seulement vécu. En d'autres termes, l'attitude critique consiste à subordonner le philosophique au religieux, le savoir à la foi, comme le remarque Hegel dans Foi et savoir. Or s'il n'y a pas de savoir de l'absolu, la philosophie ne peut être qu'une théorie du non-savoir de l'absolu, c'est-à-dire la démonstration que l'objet peut être déduit du sujet ou, en d'autres termes, le savoir de la foi, et que, tout savoir reposant sur la foi en Dieu, Dieu ne saurait être à son tour l'objet d'un savoir. Au fondement de la Doctrine de la science se trouve par suite une expérience (un fait vécu), l'expérience du religieux telle qu'elle 
se trouve thématisée dans les textes sacrés, expérience sur la possibilité de laquelle la Doctrine de la science s'interroge. On retrouve ici la question qui avait fait l'objet de la première publication de Fichte : une révélation divine est-elle possible ? La Doctrine de la science ne fait donc que reprendre cette question, mais en déduisant tout ce qui dans l'Essai d'une critique de toute révélation était présupposé. La Doctrine de la science, explique Goddard, n'a donc pas pour but de remplacer l'Évangile, comme l'ont cru certains commentateurs et contemporains de Fichte. Au contraire, dans la mesure où elle ne prétend rien faire d'autre que de déduire en pensée ce qui se trouve donné dans l'expérience, elle reconnaît n'être en mesure de reproduire que ce que les textes sacrés contiennent déjà. Cependant, comme ceux-ci sont pour le moins obscurs et sujets à interprétation, la Doctrine de la science, qui met en lumière les principes à partir desquels le discours religieux peut être déduit, indique par le fait même comment ceux-ci doivent être compris. C'est ce qui fait dire à Goddard que le rôle de la philosophie est essentiellement herméneutique.

La défiance dont on a fait preuve à l'égard de la notion fichtéenne de sujet absolu n'est donc nullement justifiée. Fichte pose en principe que le sujet est absolu afin de démontrer que l'objet peut en être déduit, c'est-à-dire que le savoir est subordonné à la foi. L'expérience du cogito, la prise de conscience de soi, n'est rien d'autre que l'expérience de la fondation de l'objet dans le sujet, c'est-à-dire du savoir dans la foi ou, en d'autres termes encore, de l'expérience que fait le sujet de son «être-créé » (p. 59), de son origine que, faute de pouvoir se l'expliquer, il ne peut se représenter que par analogie avec le divin (cf. p. 60). Loin d'être le fait d'une individualité qui nie sa finitude et se prend pour Dieu donc, le projet fichtéen invite plutôt à la reconnaissance de notre ignorance radicale et de notre statut de créature, c'est-à-dire à l'humilité.

C'est ce qui apparaît d'ailleurs avec toute la clarté voulue dans l'Initiation à la vie bienheureuse, où Fichte se réapproprie la métaphysique du prologue de l'évangile johannique qui, quoiqu'elle affirme la primauté du Fils, reconnaît la transcendance du Père (cf. p. 96). Fichte interprète ce paradoxe de la manière suivante : Dieu et l'homme sont un : dans la conscience de soi, l'homme prend conscience de son origine et de sa nature divine; mais il s'agit là d'une conscience négative : je prends conscience de moi comme étant de nature contraire à la nature de ce que je ne suis pas, c'est-à-dire comme étant de nature non-empirique ; ma véritable origine toutefois, je ne me l'explique pas, ce pourquoi je lui attribue un caractère divin ; c'est-à-dire que cette origine divine que je me reconnais, je suis forcé de reconnaître également qu'elle me dépasse absolument.

Ces considérations permettent de jeter un nouvel éclairage sur la nature du premier principe de la Doctrine de la science. Si la conscience de soi avait été conscience positive, elle aurait été conscience de la coïncidence effective du sujet et de l'objet, et l'identité du sujet et de l'objet aurait été un fait (Tatsache) : c'est la position de Schelling (cf. p. 100). La conscience de soi est toutefois selon Fichte précisément conscience négative, c'est-à-dire conscience de ceci que le sujet n'est pas objet, mais projet, non pas fait accompli (Tatsache), mais fait à accomplir (Tathandlung). En d'autres termes encore, l'identité du sujet et de l'objet ne doit pas être pensée comme chose du passé, ce qui ferait de l'état de scission actuel un état de malheur et de dégradation (c'est la conception de l'être humain exposée dans l'Ancien Testament : l'homme porte le poids d'un péché originel, c'est-à-dire que l'homme fait librement le mal), mais comme chose du futur (c'est la conception de l'être humain du Nouveau Testament : l'homme est racheté de son péché, il n'est pas radicalement mauvais). Et c'est aussi pourquoi l'action chez Schelling est posée comme étant inefficace (la condition humaine est essentiellement 
tragique : le combat pour la liberté est perdu d'avance), tandis qu'elle est posée par Fichte comme efficace (la condition de l'homme est pour ainsi dire, selon Fichte, épique) : l'homme doit joyeusement partir à la conquête de lui-même, dans la foi inébranlable que, dans l'éternité, la liberté a déjà vaincu.

La destination éthique de l'homme s'accomplit donc au moyen de l'action réciproque qu'exercent l'une sur l'autre deux tendances qui existent en lui. Selon la première, l'homme se pose comme sujet absolument libre (comme étant d'origine divine). Selon la seconde, il se pose comme limité par l'objet. La synthèse de ces deux tendances consiste dans la position de la liberté non comme effective, mais comme projet humain (cf. pp. 134-138).

Ces deux tendances toutefois sont susceptibles de "s'émanciper du jeu alterné en lequel elles se limitent l'une par l'autre » (p. 38). La première tendance prend-elle le dessus : il en résulte une perte de l'extériorité qui n'est pas sans rappeler ce que Freud a caractérisé comme état psychotique : c'est la position de l'idéaliste dogmatique qui, comme le psychotique, prétend être Dieu. La seconde tendance prend-elle le dessus : c'est alors le pouvoir de "transcender » (p. 139) l'extériorité qui est perdu, d'où résulte un état de frustration qui se compense dans un état mélancolique qui rappelle l'état névrotique : c'est la position du réaliste dogmatique qui, s'il est cohérent, est également nihiliste et nie un sens de l'existence. La voie du bonheur ou, plutôt, de la béatitude (et de la santé mentale), se trouve selon Fichte dans la mise en accord de ces deux tendances extrêmes.

Je ne prétends pas avoir épuisé par ce qui précède le contenu de l'ouvrage dont il s'agit ici. Je me suis contenté de faire surtout ressortir ce que je considère être le point d'articulation de l'ouvrage de Goddard, c'est-à-dire l'idée selon laquelle l'idéalisme transcendantal de Fichte est un moyen terme entre le réalisme dogmatique, qui réduit le moi au monde, et l'idéalisme dogmatique, qui réduit le monde au moi, de sorte que si le moi selon Fichte n'est pas néant, il n'est pas non plus l'absolu lui-même, contrairement à ce que l'on affirme sans cesse depuis maintenant plus de 200 ans, mais plutôt moyen terme entre le néant et l'absolu, c'est-à-dire une image de l'absolu.

MANUEL ROY

Freie Universität Berlin

\section{Schelling, F. W. J., Exposition de mon système de la philosophie, suivi de Schelling, F. W. J. Sur le vrai concept de la philosophie de la nature, et Fichte, J. G. Sur l'exposition du système de l'identité de Schelling, traduction et présentation par Emmanuel Cattin, Paris, Vrin, 2000, 194 pages.}

L'année 1801 aura été décisive pour l'histoire de ce qu'il est maintenant convenu d'appeler l'idéalisme allemand. Année de la parution de l'Exposition de mon système de la philosophie de Schelling, elle marque, comme le souligne Emmanuel Cattin dans sa présentation, un point tournant dans le développement de la pensée spéculative (p. 26). Cela non seulement du point de vue des commentateurs actuels. Schelling luimême, qui passait jusqu'alors pour être un simple disciple et commentateur de Fichte, exigea publiquement pour la première fois dans l'avertissement à son Exposition de 1801 « que l'on se décide [...] à considérer les expositions de Fichte et les [...] "siennes" 